\title{
Participación y Comunicación Social en Medicina Familiar y Comunitaria y Atención Primaria en Salud
}

\author{
Participation and Social Communication in Family and Community Medicine and Primary Health Care \\ Participação e Comunicação Social em Medicina de Família e Comunidade e Atenção Primária \\ à Saúde
}

\section{Coordinadores:}

Dra. Marina Almenas

Dra. Noris Serrudo

\section{Autores:}

Dra. Marina Almenas, Tesorera de la Confederación Iberoamericana de Medicina Familiar (Wonca Iberoamericana-CIMF). Pasada Presidenta de Academia de Médicos de Familia de Puerto Rico. Delegada de Puerto Rico de American Academy of Family Physician. Delegates Puerto Rico. Congress of Delegates "Reference Committee Public Health \& Science".

Dra. Noris Serrudo, Vicepresidente de la Subregión Países Andinos de Wonca Iberoamericana-CIMF. Coordinadora Regional del Programa de Medicina Familiar de la Secretaria de Salud de Gobernación de Estado Zulia, Venezuela. Secretaría de Finanzas de Capitulo Zulia de Sociedad Venezolana de Medicina Familiar (SOVEMEFA). Ex presidenta de SOVEMEFA (periodo 2006-2012).

Dra. Carmen Cabezas, Ex presidenta de la Sociedad Ecuatoriana de Medicina Familiar (SEMF). Profesora de pregrado y postgrado de la Pontificia Universidad Católica de Ecuador.

Dra. Alejandra Paulo, Medico Familiar y Comunitario en Policlínica Balneario Buenos Aires, Maldonado. UDA Rural. Uruguay.

Dr. Gustavo Montiel, Medico Familiar. Coordinador Técnico de Relaciones Públicas y Difusión del Colegio Mexicano, MF. Catedrático en la Universidad Autónoma de Coahuila. México.

Dra. Lidia G. Caballero. Médica General/Familiar. Actual Secretaria de la Federación Argentina de Medicina Familiar y General (FAMFyG) y Presidente de la Asociación Misionera de Medicina General/Familiar y del Equipo de Salud (AMMeGeFES).

Dra. Lilia Gonzalez Cárdenas: Especialista de primer y segundo grado en Medicina Familiar, Master en Educación Médica, Categoría Docente auxiliar, Categoría de Investigador agregada. Tesorera de la Junta de Gobierno Nacional de la Sociedad de Medicina Familiar en Cuba.

Dra. Andreali Morón, Especialista en Medicina Familiar, Profesora de Universidad del Zulia, LUZ, Venezuela.

Dra. Evelyn Roa, Medico Familiar. Secretaria de Publicaciones Científicas de Sovemefa. Docente Medicina Familiar, Universidad Rómulo Gallegos, Estado Guárico, Venezuela.

\section{Colaboradores:}

Dr. Rolando Herrera. Medico Familiar Policlínico Villa Galindo. Caja Nacional de Salud. Cochabamba. Presidente de la Sociedad Boliviana de Medicina Familiar (SOBOMEFA).

Dr. Alexis Correa. Medico Familiar, Miembro de junta directiva Asociación Nicaragüense de Medicina Familiar. ANIMEF. Comité de Certificación y recertificación en M.F.

Dra. Veronica Redin. Tesorera de la Sociedad Ecuatoriana de Medicina Familiar (SEMF).

Dr. Nulvio Lermen Junior. Presidente de la Sociedade Brasileira de Medicina de Familia e Comunidade (SBMFC).

Dr. Arturo Quizphe, Profesor de la Universidad de Cuenca, Ecuador. Miembro del Consejo Asesor Internacional del Movimiento por la Salud de los Pueblos. Dra. Sara Huertas, Catedrática Departamento de Psiquiatría Recinto de Ciencias Médicas, Universidad de Puerto Rico. Representante de Alianza por la Salud de los Pueblos.

Sra. Eva Tudela. Directora de la Sociedad Española de Medicina de Familia y Comunitaria (semFYC). Periodista. España.

Cómo citar: Almenas M, Serrudo N, Cabezas C, Paulo A, Montiel G, Caballero LG et al Participación y Comunicación Social en Medicina Familiar y Comunitaria y Atención Primaria en Salud. Rev Bras Med Fam Comunidade. 2016;11(Suppl 1):31-46. http://dx.doi.org/10.5712/rbmfc11(1)1279
Financiación: ninguna declarada Aprobación ética: no aplicable.

Conflicto de intereses: ninguna declarada. Procedencia y revisión por pares: revisado por pares. Recibido el: 19/02/2016. Aceptado el: 19/03/2016. 


\section{Resumen}

Se conformó el Grupo de trabajo \# 2, de la V Cumbre Iberoamericana de Medicina Familiar, Quito, Ecuador 2014. Se realizaron encuestas, entrevistas, y reuniones vía skype, internet y correo electrónico; los resultados de esta investigación fueron analizados y se presentaron en el marco de la V Cumbre Iberoamericana de Medicina Familiar. Se recopilaron todas las actividades de participación social realizadas en los países Iberoamericanos. Se pretenden establecer proyectos que fomenten alianzas y permanezcan trabajando después de la Cumbre en el tema de "Participación y Comunicación Social en MF y APS" junto a instituciones que tienen este asunto como su misión.

\section{Abstract}

Group \# 2 from the V Iberoamerican Summit of Family Medicine, Quito, Ecuador 2014 was formed. There were surveys, interviews, and reunions via Skype, internet, and e-mails and the results from this investigation were presented and analyzed in the V Iberoamerican Summit of Family Medicine, which was focused in: "Participation and Social Communication in Family Medicine and Primary Health Care".
Palabras clave:

Participación

Inclusión

Comunicación

Promoción social

Medicina Familiar y Comunitaria

Atención Primaria de Salud

Competencias

Equipos interdisciplinarios

Atención integral continua

Keywords:

Participation

Inclusion

Communication

Social Promotion

Primary Health Care

Family Medicine

Competitions

Family Physicians

Interdisciplinary Team

Continued Integral Attention

Palavras-chave:

Participação Social

Inclusão

Comunicação

Promoção Social

Medicina de Família

e Comunidade

Atençao Primária à Saúde

Equipes Interdisciplinares

Atenção integral e contínua

\section{Introducción}

En el contexto Internacional, donde los gobiernos de los países realizan transformaciones sociales, políticas y económicas, una de las mayores que buscan es en los Sistemas de Salud, de forma tal que puedan responder a las necesidades de la población. Usualmente las decisiones en los Servicios de Salud se toman desde la perspectiva de los gobernantes, sus asesores y los prestadores de servicios.

Hoy día, el termino Democracia Participativa da un giro a la forma en que se deben tomar estas decisiones en cuanto a los servicios a ofrecer; para esto debemos tener presente la Participación Social, donde se toma en cuenta la visión de los usuarios en el diseño, operación y control de Programas de Salud. La prestación de Servicios basados en las necesidades de las personas, donde el usuario también debe asumir un rol en el contexto de auto y mutuo cuidado en la salud. ${ }^{1}$

Existen una serie de principios básicos en los cuales las organizaciones relacionadas a la calidad llevan sus esfuerzos. Como una de las bases para lograr estas metas está el desarrollar y fortalecer los Sistemas de Salud basados en Esquemas en Medicina de Familia y Atención Primaria. Es por esto que en esta V Cumbre Iberoamericana de Medicina Familiar queremos desarrollar la cooperación Internacional 
mediante el intercambio de experiencias, la movilización de apoyos, la promoción de proyectos y la formación de alianzas para conseguir los objetivos propuestos. ${ }^{2}$

En las Cumbres anteriores, con el valioso aporte de una gran diversidad de profesionales de la medicina familiar y de la atención primaria de Iberoamérica, se han desarrollado argumentos, propuestas y recomendaciones que de alguna forma inician el tema que este Grupo 2 aborda en este evento, tal es: "La participación y Comunicación Social en la Medicina Familiar y Atención Primaria de Salud”. En los siguientes párrafos, haremos referencia a las Cartas de Sevilla (España), Santiago de Chile, Fortaleza (Brasil) y Asunción (Paraguay), destacando las ideas que consideramos de mayor relevancia para nuestro trabajo.

I Cumbre Iberoamericana de Medicina Familiar, Declaración de Sevilla, Mayo de 2002, bajo el tema: "Comprometidos con la Salud de la Población". ${ }^{3}$

Durante los últimos años se han ido imponiendo como un sentimiento unánime la necesidad de centrar la organización en el ciudadano/a; es decir, identificar la manera en que los sistemas de salud de los países pueden responder mejor a las necesidades y demandas de los individuos, sus familias y las comunidades.

Los actuales sistemas de salud precisan reformas centradas en la salud de las personas encaminadas a mejorar la accesibilidad, la cobertura, el nivel de calidad de la atención y la satisfacción de los ciudadanos/as. En esta estrategia es esencial el papel del Médico de Familia.

Al reflexionar sobre las aportaciones de la Medicina Familiar a la mejora de la calidad de los servicios de salud, los participantes en la Cumbre de Sevilla, plantearon algunas recomendaciones que se citan en los siguientes párrafos.

Los escenarios que viven los sistemas de salud de la mayor parte de los países iberoamericanos obligan a la búsqueda de mejores instrumentos para mejorar la gestión, alinear la asignación de recursos con los procesos de mejora continua de la calidad de los servicios de salud aplicando los incentivos apropiados. Esta debe ser una tarea prioritaria de los gobiernos y organizaciones de la salud de los países de la región a la que el CIMF-WONCA y la OPS-OMS pueden aportar su capacidad técnica y abogacía institucional. ${ }^{2}$

Es necesario trabajar en modelos que se orienten a las particularidades de la atención primaria en donde el Médico de Familia, junto a los demás miembros del Equipo de Atención Primaria, es la puerta de entrada al sistema. Por ello se propone trabajar en una iniciativa regional para el fomento y desarrollo de unos modelos innovadores de Atención Primaria con base poblacional claramente definida.

Los médicos de familia son profesionales clínicos y de salud pública que trabajan en el ámbito de la atención primaria. Como tales forman parte de un equipo de profesionales de la salud, cada uno con competencia e incumbencias específicas. El trabajo interdisciplinario coordinado es una de las mayores fortalezas de los equipos de salud familiar. Cualquier iniciativa de mejoramiento de la Calidad desde la perspectiva de la Medicina Familiar debe considerar la participación activa de todos los profesionales del equipo de Salud Familiar. ${ }^{4}$

Il Cumbre Iberoamericana de Medicina Familiar, en Santiago de Chile, Octubre 2005. Tema: "El Médico de Familia garantía de calidad y equidad en los Sistemas de Salud de Iberoamérica". ${ }^{5}$

La Medicina Familiar, como especialidad orientada hacia la atención integral y continua, la promoción de la salud y prevención de enfermedades, así como la prestación de servicios en el primer nivel de atención, es el actor más apropiado para realizar las intervenciones médicas necesarias en la estrategia de la Atención Primaria de la Salud. 
Deben desarrollarse métodos y medidas uniformes para evaluar el desempeño de los sistemas de atención primaria. Estas medidas deberían incluir costo, calidad, acceso y satisfacción tanto del paciente como del médico y el equipo de salud. Los resultados debieran también estar disponibles para los financiadores, los prestadores y el público en general.

Se deben estimular las sinergias entre el sector público, la seguridad social y el sector privado para aumentar críticamente la proporción y el número de médicos de familia en relación al número total de médicos. ${ }^{6}$

III Cumbre Iberoamericana de Medicina Familiar en Fortaleza, Brasil, Abril 2008. "Misión y Desafíos para la Medicina Familiar y la Atención Primaria en el siglo XXI: "Equidad, Integralidad y Calidad para nuestros sistemas de Salud". ${ }^{7}$

Este evento, tuvo como objetivo contribuir al fortalecimiento de las políticas de salud en el campo de la Atención Primaria y la Medicina Familiar en Iberoamérica.

Para la inserción y valorización de la medicina Familiar en las políticas de salud es necesario: Compilar las evidencias existentes y generar nuevas evidencias sobre las ventajas de la especialidad. Para eso, es fundamental el papel de las sociedades científicas, universidades y otras organizaciones relacionadas en reconocer la importancia y la influencia de la Medicina Familiar en las políticas de salud orientadas a la Atención primaria en salud, APS. ${ }^{8}$

IV Cumbre Iberoamericana de Medicina Familiar en Asunción, Paraguay, Noviembre 2011. "Medicina Familiar y Atención Primaria en Salud Renovada: Pensamiento y Acción en beneficio de la Salud Familiar". 9

Reafirmar la salud como un derecho, reconocer la complementariedad de la Medicina Familiar y la APS como estrategias para el desarrollo de los sistemas de salud, en el marco de un modelo de desarrollo que busca el bienestar de las poblaciones y maximiza el potencial de las personas, se recomienda la puesta en práctica de una herramienta que mida de manera objetiva y permita realizar comparaciones entre los diferentes países de la región, tendientes a proponer estrategias y acciones, con el fin de mejorar la calidad de atención en medicina familiar. ${ }^{10}$

Se propone que esta herramienta enfocada a evaluar la actividad del médico familiar, en los principales problemas de salud y de demanda en medicina familiar, contemple indicadores en los siguientes dominios: Capacitación científico - técnica, efectividad de las acciones realizadas en promoción y prevención de la salud, orientadas al individuo, familia y comunidad, atención integral, longitudinal y continua, cumplimiento de los derechos de los pacientes y satisfacción. ${ }^{11}$

\section{Objetivos}

1. Identificar y establecer proyectos colaborativos con instituciones públicas y privadas que tengan como una de sus misiones la inclusión y participación social.

2. Apoyar la participación de las Sociedades de MF con otras Asociaciones Comunitarias.

3. Establecer actividades exitosas, pre-establecidas en algunos países que podrían ser implementadas en otros países de la región.

4. Impulsar la creación de medios escritos, radiales, televisivos, folletos, videos u otros para la difusión de la MF. 
5. Establecer la participación de la MF en las Actividades Comunitarias en todas las Regiones de Iberoamérica.

6. Llevar el mensaje de que es la Especialidad de Médicos de Familia a todas las comunidades y todos los niveles sociales y políticos.

7. Promover la creación de clínicas de Educación y Prevención con el apoyo de Educadores en Salud y Líderes Comunitarios.

\section{Metodología}

Se conformó el Grupo de trabajo \# 2, de la V Cumbre Iberoamericana de Medicina Familiar. Se recopilaron todas las actividades de participación social, realizadas en los países que componen la Confederación Iberoamericana de Medicina Familiar, mediante encuestas, entrevistas personales, por via skype y por correos electronicos, a todas las Asociaciones de Medicina Familiar de los diferentes países de Iberoamerica. Se analizaron los resultados de esta investigación, en reuniones virtuales y personales en la precumbre y durante la $\mathrm{V}$ Cumbre y se presentaron en un taller en el marco de la V Cumbre Iberoamericana de Medicina Familiar, Quito, Ecuador 2014, enfocándose en: “La Participación y Comunicación Social en Medicina Familiar y Atención Primaria de Salud".

Descripción de la Situación Actual

\section{Argentina}

El área de Docencia de la Federación Argentina de Medicina Familiar y General (FAMFyG) tiene a su cargo el desarrollo de cursos y programas que brinden a los trabajadores de la APS herramientas e instancias de formación, capacitación y actualización en contenidos relacionados con sus núcleos de competencia.

Está integrado por un cuerpo docente de destacados profesionales en cada área temática, que tienen como norte el promover una práctica de calidad, basada en la equidad. El área de Docencia organiza programas tanto presenciales como a distancia, para lo cual cuenta con un Aula Virtual y toda la tecnología actual en educación y capacitación en salud.

El actual proceso de certificación fue diseñado teniendo en cuenta estas realidades. Se trata de convocar a todos los profesionales que acreditando ciertas experiencias curriculares (créditos) y que tengan una práctica afín a la Medicina Familiar y General puedan acceder al examen de certificación. La convicción de esta Federación reevaluar continuamente el proceso de certificación para alcanzar los estándares superiores que se esperan de un Médico General y de Familia.

Archivos de Medicina Familiar y General es una publicación dirigida a los equipos de salud, profesionales en general y estudiantes, que propone la reflexión sobre la práctica, informa sobre nuevos conocimientos y nuevas estrategias para el trabajo del equipo, muestra producciones locales, regionales e internacionales, actualizaciones clínicas, recupera experiencias en terreno, analiza teorías vigentes. Todas estas áreas se centran sobre los tres ejes que propone la línea editorial: la educación, la micro política de trabajo y la clínica ampliada. 


$\begin{array}{ll}\text { Países } & \text { Situación Actual en su país a base de la "Participación y Comunicación social para la Medicina Familiar y Comunitaria } \\ \text { y la Atención Primaria en Salud }\end{array}$

Se llevan a cabo encuentros comunitarios con la participación del Equipo de Salud y la Comunidad. Estas actividades son $\begin{array}{ll} & \text { realizadas por las distintas asociaciones locales. También se utilizan los medios de comunicación digital: www.famfyg.org } \\ \text { Argentina } & \text { Facebook: FAMFYG, escrita: Revista Archivos de Medicina Familiar y General, televisiva y radial. Congreso Nacional de }\end{array}$ la especialidad anual dirigido a Médicos de Familia/Generales y al Equipo de Salud, rotando en las diferentes provincias y regiones del país.

Se realizan actividades educativas mediante charlas en salas de espera, reuniones de clubes y organizaciones territoriales de base de clubes en las policlínicas de la seguridad social respecto a la mejora del estilo de vida, prevención de

Bolivia enfermedades prevalentes con participación activa del equipo de salud familiar. Se realizan visitas domiciliarias lideradas por médicos familiares, se llevan a cabo Ferias de Salud en parques, y plazas. Otro emprendimiento interesante es la vigencia del Programa de Radio, Revista Impresa y Televisión denominado; "Enfoque a la Salud" realizado por Médicos Familiares; programa exitoso que acaba de cumplir 25 años de vigencia. (Ver en blog de Facebook Enfoque a la Salud Bolivia)

Utilizan la radio, prensa escrita, tv. e internet para la difusión y educación de la MF. Como actividades comunitarias algunas asociaciones estaduales vinculadas a la Asociación Nacional (SBMFC) tienen actividades, pero no podemos decir que hay algo organizado para todo el país.

El Estado desarrolla un proceso de fortalecimiento, para acercar cada vez más a la población, a la solución de los problemas

Cuba de salud, mediante la concertación, negociación y participación social y comunitaria. Se realizan charlas como tipo de actividades comunitarias y se utilizan los medios tecnológicos para difusión.

Ecuador Como estrategias de comunicación existen folletos para el embarazo, nutrición; además contamos con radio web "Radio Play Internacional", Web: www.semf.ec y Facebook y twitter donde promocionamos actividades educativas.

Como tipo de actividades sociales y comunitarias se realizan certámenes. Para la difusión de la especialidad se hacen España actividades comunitarias, además se utiliza la prensa escrita; Revista Comunidad, Revista AMF, Revista de Atención Primaria. La Obra editorial de SemFYC. http://www.semfyc.es/es/publicaciones/ultimas/. http://www.semfyc.es/es/biblioteca/virtual/.

Se realizan charlas, certámenes y actividad comunitaria. En México afortunadamente la certificación de la especialidad México es ya obligatoria cada 5 años, actualmente las buenas relaciones entre el Consejo y el Colegio Mexicano de Medicina Familiar, nos permiten unificar fortalezas para influir en los Médicos Familiares durante su capacitación para la certificación y en las reuniones de Congresos.

Desde 2007 ha sido poca la participación de los Médicos Integrales o Familiares, gobierna el desinterés y en muchas ocasiones la falta de tiempo... antes eran reuniones llamadas encuentros bi-anuales con la finalidad de presentar temas

Nicaragua de interés médico. Pienso en futuro crear un grupo que evitando los peros de ni poder reunirnos al promover utilizar la tecnología moderna para a través de internet hacer un grupo de trabajo aún en proceso de confirmar el número y quienes protagonizarán.

Realizan actividades sociales y comunitarias tales como: charlas, clínicas y certámenes. Para la difusión de la especialidad

Puerto Rico MF se utiliza la radio, prensa escrita, tv., internet www.amfpr.org, cine, redes sociales twitter, Facebook, Revista AMFPR y actividades comunitarias. Contamos con Video de MF de 30 segundos y video para actividades de 4 minutos.

Actividades intersectoriales: fundamentalmente con el sector educación. Cursos de agentes comunitarios de salud (ACS) en varios puntos del país, organizados y coordinados por los propios MFYC. Campañas a nivel nacional sobre diferentes temas a saber: salud cardiovascular, salud de la mujer, violencia doméstica, hipertensión arterial, SIDA, lactancia materna,

Uruguay salud ocular, salud mental, salud renal, bucal, etc. Conformación de Unidades Docentes Asistenciales de MFC en la capital del país y varios departamentos del interior incluyendo el área rural de MFYC. Participación en mesas intersectoriales zonales Participación en programas de radio sobre salud y video conferencias. Programas televisivos donde exponemos sobre actividades de promoción o prevención y sobre nuestro perfil.

Se llevan a cabo charlas, sesiones educativas tipo talleres y actividades extramurales: Operativos médicos o clínicas.

Venezuela Como medios de comunicación se utiliza la prensa escrita, radio, internet, videos, redes sociales, fotografías, carteleras, folletos, afiches.

Archivos de Medicina Familiar y General integra el Núcleo Básico de Revistas Científicas Argentinas, dependiente del Consejo Nacional de Investigaciones Científicas y Técnicas (CONICET) y ha sido indizada en su catálogo (LATINDEX) con resultado NIVEL 1 (nivel superior de excelencia).

Biblioteca de la Federación: Los diferentes recursos que la FAMFyG pone a disposición, algunos de producción propia, otros seleccionados de otros sitios y organizaciones que se relacionan con la Atención Primaria de la Salud. 
Entre otros, se dispone de:

- Documentos de posición de la FAMFyG y otros documentos de interés en APS

- Fotos de eventos de la FAMFyG y relacionados.

- Videos institucionales, didácticos y de utilidad para el Equipo de Salud

- Recursos multimedia

- Presentaciones de clases y conferencias en PPT y PDF de libre acceso.

- Enlaces de utilidad, páginas de interés, bases de datos, etc.

\section{Bolivia}

En Bolivia la Comunicación Social es una necesidad imperiosa pero los esfuerzos de los médicos familiares que trabajan en comunicación interpersonal y a diario no tienen el apoyo gubernamental.

El gobierno actual que en teoría es un gobierno de transformación social, política y de universalización y accesibilidad a la salud se encuentra absolutamente divorciado del ente colegiado, el Colegio Médico de Bolivia y por ende de las células de esta organización, una de las más importante es la Sociedad Boliviana de Medicina Familiar.

Actualmente el gobierno solamente apoya acciones del modelo denominado Salud familiar Comunitaria e Intercultural con un evidente tinte ideológico político y la presencia de médicos cubanos o médicos bolivianos formados en Cuba, que no gozan de la confianza de la población y las comunidades, ahondando cada vez más la brecha ente los médicos familiares y los denominados médicos SAFCI, que prácticamente han rechazado los esfuerzos de acercamiento establecidos por la Sociedad Boliviana de Medicina Familiar.

Otra de las transformaciones importantes es la visita a Empresas donde se informa, educa y se realiza revisiones médicas tipo screening o chequeos médicos programados, inmunizaciones y detección de factores de riesgo a obreros y empleados que trabajan en empresas situadas en zonas de adscripción de los médicos de familia.

No obstante, los médicos familiares realizan actividades continuas y programadas mediante charlas en salas de espera, visitas a domicilios e instituciones y en los últimos cinco años las exitosas Ferias de Salud en plazas, parques o centros educativos.

\section{Cuba}

El surgimiento de la Participación Comunitaria como categoría social es muy antigua, tanto como los primeros grupos sociales, pero desde la perspectiva del desarrollo comunitario es mucho más reciente. Nosotros tenemos un modelo de atención con un Programa del médico y enfermera de la familia que en Cuba tiene 30 años de creado desde 1984. Este modelo se caracteriza en su concepción por una voluntad política basada en el trabajo en equipo de un médico y una enfermera, que brindarían atención médica integral, dispensarizada y proactiva a un sector de la población, y que consideraban a la familia como la unidad principal de atención, aunque también realizaron acciones sobre el individuo y la comunidad donde radica, para el trabajo de este Médico y Enfermera de Familia es necesaria la participación activa de la comunidad y la intersectorialidad en la gestión de salud, con el propósito de promover la salud a través de cambios positivos en los conocimientos, hábitos de vida y costumbres higiénico-sanitarias de la población, 
prevenir la aparición de enfermedades y daños en la salud de la población, garantizar el diagnóstico precoz y la atención médica integral, desarrollar la rehabilitación con base comunitaria dirigida a la población discapacitada física o psíquicamente, alcanzar cambios positivos en el saneamiento ambiental de su radio de acción, así como de las condiciones higiénicas en que viven las familias bajo su control, y desarrollar investigaciones que respondan a las necesidades de salud de la comunidad.

La comunidad cubana tiene un peso importante como escenario de la participación popular, participación entendida en su sentido más integral: la intervención activa en todo el proceso social, desde la identificación de problemas, necesidades, definir formular políticas, ejecutarlas y controlar el desarrollo de la actividad en torno a dichas políticas.

\section{Ecuador}

En Ecuador la existencia de la Medicina Familiar se inició hace aproximadamente 25 años con pocos especialistas que hicieron sus estudios en México y Estados Unidos. Es hace tres años que el Ministerio de Salud Pública reconoce como especialidad a la Medicina Familiar y crearon las plazas para especialistas y los médicos familiares que trabajaban como Médicos Generales fueron reconocidos en toda su implicación (remuneración, responsabilidad y trabajo).

La participación comunitaria de la Medicina Familiar en el país fue un asunto aislado, individual y por la iniciativa única de unos pocos que querían hacer algo en su espacio. Desde los espacios docentes de formación, que desde hace 15 años han existido, había alguna participación y comunicación social en espacios cerrados privados y particulares a través de vínculos débiles como comunidades religiosas y en espacios públicos, como subcentros de salud de primer nivel. En estos pequeños espacios se realizaba educación a la comunidad contribuyendo a solventar las necesidades puntuales de cada una sobre todo en actividades de prevención y promoción como charlas.

Debemos dejar en claro, que si ha habido participación y comunicación social y Atención primaria en salud desde el estado ecuatoriano con diferentes programas implantados desde el ministerio de Salud Pública que dependiendo de la tendencia del gobierno han ido cambiando sin llegar a ser políticas de estado. Es hace siete años de (lo que se le llama el cuarto poder: la ciudadanía) y donde además ha habido un proceso de reconocimiento a la especialidad como instrumento clave en la APS.

Como estrategias de comunicación desde la SEMF, tenemos el Proyecto "Salud de Altura" que es una propiedad intelectual de la SEMF desde hace 6 años, a través de los cuales pudimos publicar y difundir principalmente tres productos (libros): Relación Médico Paciente, Toma de decisiones Clínicas, Vademecum actualizado hasta el 2011 y además Folletos preparatorios para el embarazo, Folletos para acompañar en la nutrición que son ofrecidos en la SEMF a los postgradistas y la comunidad.

Tenemos oficialmente hace tres meses un programa de radio en Radio Play Internacional, la cual es la primera radio Web ecuatoriana y está a disposición internacionalmente para todos ustedes. Se debe recalcar que el programa está dirigido y producido por médicos de familia de la SEMF y se debate sobre temas absolutamente de nuestra especialidad todos los martes.

Además disponemos de lugar Web, Facebook y twitter donde constantemente promocionamos actividades educativas, políticas y sociales, vinculadas con actividades donde participen los médicos de familia en el país. 
El espacio Web: www.semf.ec tiene aula virtual con educación en línea, espacios de discusión académica, links de interés y sobre todo la oferta docente desde la SEMF al público en general de curso puntuales como: Gestión de Salud, Medicina Basada en Evidencia, Toma de decisiones Clínicas e Instrumentos en la APS.

\section{México}

En México, la Medicina Familiar tiene presencia en las principales instituciones de salud pública, sin embargo la difusión sobre las bondades de la especialidad no son del todo óptimas, ya que la especialidad fue creada para dar respuesta a las crecientes necesidades de una mejor y sistematizada atención para la población asegurada como beneficio de la seguridad social, creada para los trabajadores y sus familiares. El enfoque generalista que se ha dado en el primer nivel de atención no les permite a los pacientes distinguir la realidad de la especialidad.

Es común que algún paciente le pregunte al médico de familia ¿Cuál es la diferencia del médico familiar y el médico general?, ya que de manera indistinta le atiende uno u otro en el primer nivel de atención médica; de hecho el motivo de la pregunta es porque la percepción de atención es diferente en la gran mayoría de los casos, sin embargo, se desenvuelven en la misma unidad de atención médica que Ilaman Unidad de Medicina Familiar. Al no existir un número suficiente de médicos especialistas en Medicina Familiar para dar respuesta a las necesidades de atención a la población, algunos consultorios de las unidades de primer nivel son ocupados por médicos generales.

Las instituciones cumplen con la atención médica, pero no se preocupan por difundir que sus médicos son especialistas en Medicina Familiar, de tal forma que los pacientes en su mayoría no saben distinguir las características propias del Médico Familiar, y la mayoría piensa que solo en los hospitales se encuentran los especialistas, pues es a donde se les envía para ser intervenidos quirúrgicamente o a ser hospitalizados para resolver su problema de salud, y que es donde se encuentran la mayoría de los recursos tecnológicos con los que se llega a la mayor precisión diagnóstica.

El desconocimiento de las características y experticias de los Médicos de Familia especialistas se extiende entre otros médicos especialistas, olvidan que el Médico Familiar, como primer contacto, no solo promueve acciones de educación para la salud, protección específica, detección oportuna de enfermedades, tratamiento inicial con la intención de limitar el daño. Además, dichas acciones serán útiles en la posterior valoración que ellos realicen para evitar posibles complicaciones, usando recursos técnicos con los que el primer nivel de atención no cuenta.

También el Médico Familiar desempeña un papel importante en la rehabilitación del paciente, pues después de ser atendido a nivel hospitalario el paciente regresa al consultorio de Medicina Familiar para continuar su tratamiento. Esto pone de manifiesto que el Médico Familiar tiene un enfoque holístico de la atención de la salud, pues interviene en todos los niveles de prevención de la historia natural de la enfermedad.

Otro punto que requiere fortalecimiento, dada la poca información sobre la Medicina Familiar está en las escuelas y facultades de Medicina, ya que la mayoría de los jóvenes médicos optan por otras especialidades. Esto es debido a que sus profesores los deslumbran con la relatoría de casos de pacientes hospitalizados, pero que en el contexto de la atención a las demandas del servicio médico representan del 
12 al 15\% y de esos casos el 3\% será enviado a un tercer nivel de atención, donde los subespecialistas tienen aún más y mejores recursos técnicos con los cuales deberán resolver la problemática del paciente.

Este año gracias al entusiasmo y participación activa del Presidente del CMMF, Dr. Homero De los Santos Reséndiz, se reactiva un importantísimo órgano de difusión del Colegio Mexicano de Medicina Familiar como es la Revista del propio Colegio. La cual, permitirá mantener informados a los Médicos Familiares sobre acciones de orden internacional que deberán acrecentar el orgullo de pertenencia al Colegio, al CIMF y a WONCA.

\section{Puerto Rico}

En Puerto Rico desde que comenzó la formación en el Programa de Residencia para obtener la Especialidad Médica, se tiene como parte de su currículo la Medicina comunitaria. Esta área se lleva a cabo a veces en conjunto con la Academia de Médicos de Familia de PR. donde se organizan actividades o talleres en las comunidades de acuerdo a las necesidades de esta. Algunas de las actividades se realizan en conjunto a otras Asociaciones o Entidades que tienen dentro de sus objetivos la Participación y Comunicación social. Se utilizan diferentes técnicas; ya sean clínicas, talleres de educación según necesidades que las comunidades han expresado.

También a través de Certámenes escolares donde se desarrolla un Tema de Salud como la obesidad, el tabaquismo u otros, dando la oportunidad a las comunidades escolares desarrollar el tema en forma artística. Hace varios años en PR., existe una entidad llamada; Agenda Ciudadana la cual tiene una Integración Multisectorial, ellos han desarrollado el Concepto de Participación y Comunicación Social a través de toda la Isla; creando estrategias propuestas por la misma comunidad y sus líderes comunitarios en básicamente todos los sectores, incluyendo la Salud de forma tal que es la misma comunidad quien informa sus necesidades reales.

La AMFPR continua promocionando la Especialidad de Medicina de Familia, como el Médico que une todas las especialidades y lo más importante, es el Aspecto Bio-sicosocial, concepto con el cual se logra una verdadera educación y prevención a la población y sus comunidades. El Departamento de Salud también realiza numerosas actividades de educación a las comunidades.

La Alianza de Salud para el Pueblo (Alianza) se constituyó en 2011 mediante un acuerdo de colaboración entre 4 organizaciones:

Entre los principios de nuestra organización, más relevantes de la medicina de familia y comunitaria están los siguientes:

1. Promover un sistema que integra los servicios con un sistema de cuidado primario fortalecido.

2. La atención directa de los individuos y la atención a las condiciones sociales que determinan la salud y las intervenciones que promueven la salud y previenen la enfermedad.

3. Un sistema que eduque y promueva que los participantes del sistema y la ciudadanía en general asuma responsabilidad social y participe en todos los niveles del sistema, desde el cuidado de la salud propia y el ambiente hasta la gobernanza del sistema.

Estos principios y propuestas han sido divulgados a través de los medios de comunicación tales como programas radiales, televisión y prensa escrita. Además se han presentado en discusiones 
directas con diversos sectores comunitarios, incluyendo profesionales de la salud, sindicatos, líderes comunitarios y religiosos, universidades y en actividades de la Fundación Agenda Ciudadana. Dicha fundación promueve la participación ciudadana a través de foros comunitarios de diversos temas en todo el país. Por muchos años y de diversas maneras dicha organización ha promovido la discusión y formulación de recomendaciones ciudadanas a diversos problemas en diferentes áreas, incluyendo salud, educación y economía, entre otras.

\section{Uruguay}

La difusión de la especialidad la hacemos a través de actividades intersectoriales: fundamentalmente con el sector educación: se realizan talleres en Escuelas, Liceos, Centro de Atención Inicial a la Familia (CAIF) centros de Apoyo Escolar y liceal. En ese sentido aprovechamos instancias para difundir aspectos que hacen a nuestro rol como MFYC.

Se realizan cursos de agentes comunitarios de salud (ACS) en varios puntos del país, organizados y coordinados por los propios MFYC orientados a la democratización del conocimiento sobre derechos ciudadanos, participación comunitaria, estilos de vida saludables, comunicación, planificación local participativa, entre otros temas también nos permite trabajar en base a una modalidad que muestra cual es nuestra propuesta pero también nos permite incorporar a los agente comunitario en salud, ACS en los equipos de trabajo y mediante ellos difundir nuestro rol. El rol del agente comunitario en salud ha tomado fuerza en nuestro país y es importante trabajar con ellos en la difusión de nuestro rol, ellos conocen la realidad de su propia comunidad y lógicas de comunicación que manejan sin duda mejor que nosotros.

Durante el año se realizan a nivel nacional campañas sobre diferentes temas a saber: salud cardiovascular, salud de la mujer, violencia doméstica, hipertensión arterial, SIDA, lactancia materna, salud ocular, salud mental, salud renal, bucal, etc. Generalmente los días específicos que conmemoran aspectos de la salud se realizan jornadas en varios puntos del país en simultáneo instancias que sirven y son utilizadas para colectivizar aspectos de nuestra especialidad.

Conformación de Unidades Docentes Asistenciales de MFC en la capital del país y varios departamentos del interior incluyendo el área rural (convenio Facultad de Medicina ASSE (Administración de Servicios de Salud del Estado) de MFYC, además de otros profesionales, esto permite el trabajo interdisciplinario y el intercambio permanente de otros técnicos que van conociendo nuestra especialidad, pero también permite aterrizar proyectos que tiene que ver con las estrategias y aptitudes del MFYC.

Estas UDAS se conformaron entre otros motivos, para ser el pilar de la formación de los estudiantes de medicina de pregrado, ya que el Nuevo Plan de Estudios de la Facultad de Medicina de la Udelar basa su formación en los primeros años en el espacio comunitario y del primer nivel de atención. Capacitación de las UDAS en la aplicación de herramienta PCAT para evaluar atributos de la APS en sus propios servicios integrados por MFYC.

Además de Montevideo los posgrados, los residentes de MFC, también realizan su formación en el interior del país, incluso en áreas rurales, esto aporta a la diversidad en su formación, pero también da la oportunidad de conocer aspectos comunitarios específicos de su lugar de trabajo y también esto permite ir creando espacios de trabajo desde la especialidad y fortalece equipos con esta mirada. 


\section{Venezuela}

Realizamos sesiones educativas tipo charlas y talleres sobre las patologías relevantes a nivel nacional, tales como diabetes, hipertensión, obesidad, y sobre problemas psicosociales como embarazo a temprana edad, sexualidad responsable y otras. También se practican talleres a nivel de las escuelas y liceos sobre temas como alimentación saludable, promoción de hábitos saludables, entre otros.

En cuanto clínicas aquí se llaman Operativos médicos, se trata de actividades extramurales, coordinadas para incrementar los beneficiarios de procedimientos como citologías, vacunación, higiene dental, aplicación de anticonceptivos, entre otros. Por lo regular estos operativos son menos frecuentes (uno o dos por año) lo más frecuente son las sesiones educativas y las visitas a centros educativos.

En Venezuela se encuentran entidades públicas como el ministerio de salud, el seguro social, estatal PDVSA, (Petróleos de Venezuela), que realizan acciones locales con los recursos humanos formados en medicina familiar que dan servicios médicos. También apoyan en estas funciones las universidades, que son sede de los postgrados de medicina familiar, en particular la Universidad del Zulia, la Universidad de los Andes en el estado Mérida, la Universidad Francisco de Miranda en el estado Falcón, y la Universidad Lisandro Alvarado en el estado Lara. Gracias a los médicos residentes de postgrado se desarrollan gran parte de las acciones educativas y algunas inclusive se evalúan en sus resultados o impacto.

En cuanto a las de carácter privado contamos con la FUNDACIÓN VENEZOLANAPARALAMEDICINA FAMILIAR, que tiene entre sus objetivos promocionar la especialidad a nivel comunitario. Una reciente incorporación de apoyo a la medicina familiar es la empresa privada prestadora de servicios de salud llamada: RESCARVEN. Finalmente hay una asociación civil comunitaria que inclusive publica en varios diarios de circulación nacional que siempre han apoyado a la Medicina Familiar, interactuando con FUNVEMEFA.

Las actividades se fundamentan en acciones preventivas, charlas grupales a pacientes en sala de espera con temas de interés relacionados con la enfermedad, entrega de trípticos educativos y charlas individualizadas a cada paciente en su consulta. Las actividades de la consulta no solo están confinadas a nuestra área dentro del ambulatorio, las acciones preventivas también salen a la comunidad, principalmente en alianza estratégica con las escuelas del área de influencia.

Existen los Club de Diabetes que se reúnen una vez al mes y está conformado por personal médico, de enfermería, de nutrición y de trabajo social, con pacientes diabéticos y sus familiares, así como con personas sanas con riesgo de tenerla, con la finalidad de ofrecer educación permanente, oportuna y de calidad, para motivar y sensibilizar sobre los hábitos de Vida Saludables como herramientas de prevención, control y tratamiento de la Diabetes Mellitus tipo 2.

El equipo de salud liderizado por el médico de familia se reúne previamente para la planificación, selección de los temas a tratar y las estrategias a utilizar. La promoción de las actividades tiene el apoyo del servicio de trabajo social y se utilizan los contactos telefónicos y mensajes de texto como recordatorios de las actividades.

\section{Conclusiones}

A continuación se presentan las diferentes actividades y/o proyectos de Participación Social para Medicina Familiar y Comunitaria y Atención Primaria en Salud; que se lograron identificar y fueron suministradas por los representantes de cada país que participaron durante la cumbre. 
1. Reuniones con las comunidades para conocer sus necesidades y prioridades en salud y formulación de planes.

2. Acompañamiento a grupos de agentes comunitarios en articulaciones intersectoriales.

3. Proyectos Comunitarios - Certámenes, Clínicas de Salud o Día Familiar.

4. Educación a través de radio, televisión, periódicos, página web.

5. Encuentro a Nivel Nacional de Agentes Comunitarios eso tal vez permitiría generar un espacio donde podemos también difundir nuestro rol como Especialistas en Medicina Familiar y Comunitaria.

6. Actividades:

- Hacer por lo menos 1 vez al año una jornada de sensibilización de nuestros propios equipos de trabajo para que conozcan el rol del Médico de Familia y la Participación Comunitaria y Social.

- Realizar jornadas en la comunidad simultáneamente con varios países, donde se difunda claramente nuestro rol, en afiches, panfletos, boca a boca. Establecer o seleccionar el día de los Médicos de Familia y Comunidad; podría ser también en alguna jornada temática en relación a un tema de salud, incluso ese mismo día traer medios de comunicación a cada comunidad, para difundir nuestro rol especifico y continuar conociendo las necesidades de la comunidad.

7. Vídeo: Para difundir el Rol del Médico de Familia en la Atención Primaria. Disponible en: http:// youtu.be/7BRezc0CNwl

8. Intercambio de Experiencias Nacionales e Internacionales.

9. Participación en Actividades Internacionales, en los Congresos Locales, Regionales o Nacionales de Médicos Especialistas en Medicina de Familia, solicitar que cada uno de los presentes en reuniones Internacionales, inviten por lo menos a uno más a participar activamente para que se incremente la participación y de esta forma se fortalezca la Medicina Familiar. A mayor cantidad de médicos reunidos mayor fuerza y proyección de la Medicina Familiar.

10. Redes Sociales tales como: Facebook, Twitter, como Medio de Difusión y Campaña de Educación y Prevención de forma tal que se mantengan canales de comunicación.

11. Crear Grupos que evalúen y diseñen acciones dirigidas a las áreas de comunicación que mencionamos en las Estrategias, tales como: Comunicación Institucional, Relaciones Políticas, Personas Claves en Redes Sociales, Grupo de Portavoces, Estudios, Encuestas.

12. Integrar la participación y comunicación social al sector académico desde el pregrado al postgrado.

13. Poder de decisión en los Sistemas de Salud - Crear legislación.

14. Fomentar el Empoderamiento de las Personas y Comunidades.

15. Desarrollar el intercambio de ideas y proyectos con entidades y fundaciones; entre las cuales ya se encuentran: Organización Rescarven, Caracas, Venezuela. Agenda Ciudadana, Puerto Rico, Alianza por la Salud del Pueblo, PR. y el Movimiento por la Salud de los Pueblos.

16. Folleto - Educativo sobre: La Participación Social y el Médico de Familia. 

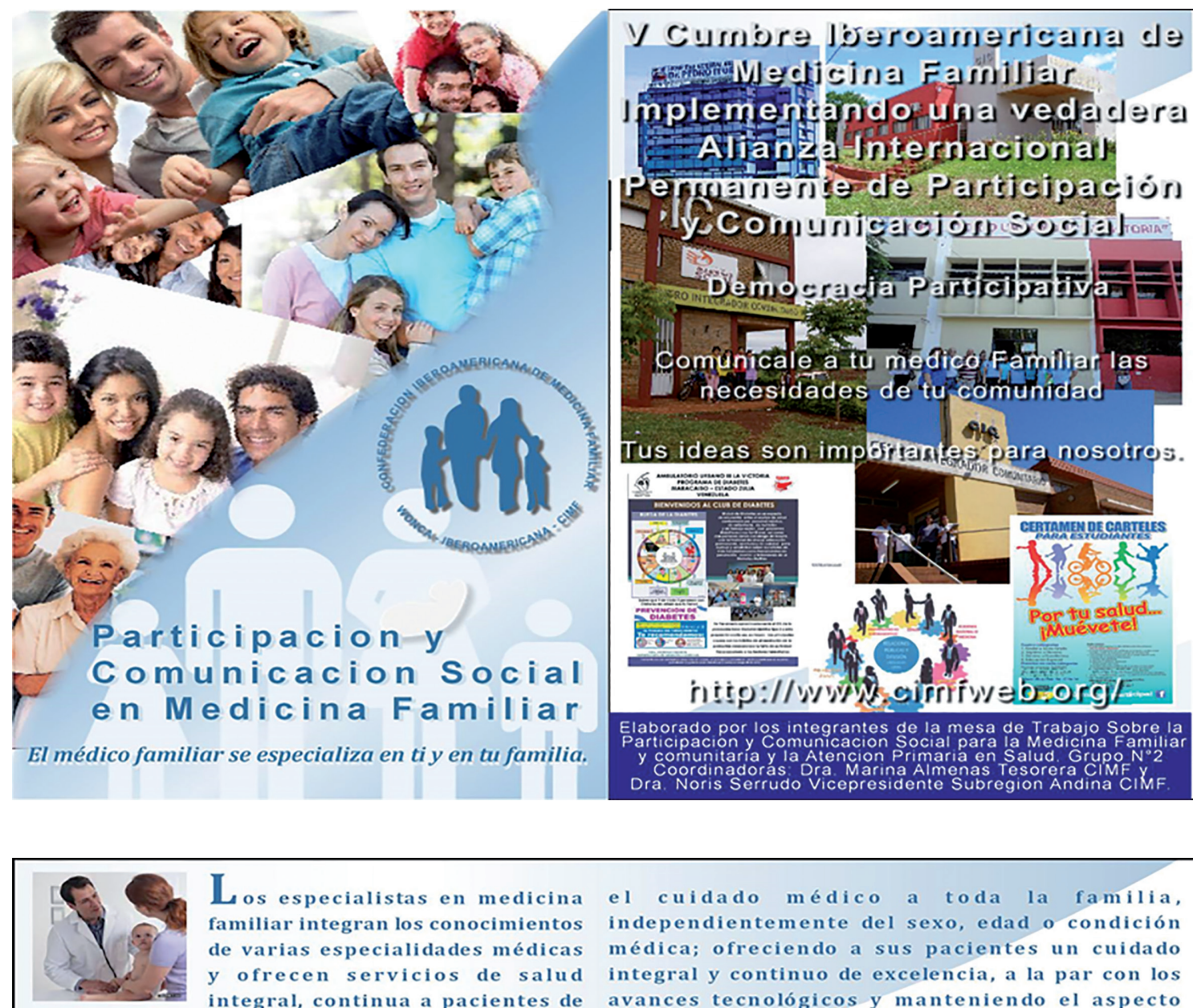

Los especialistas en medicina familiar integran los conocimientos de varias especialidades médicas $y$ ofrecen servicios de salud integral, continua a pacientes de todas las edades, sexo y condición, cuidando de la salud del paciente durante el transcurso de nuestras vidas.

El medico familiar luego de cursar estudios de medicina, realiza el postgrado de la especialidad en

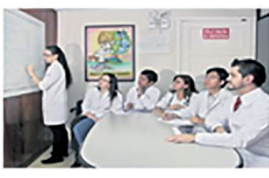
medicina familiar, con una duración promedio de tres años, adquiere conocimientos de otras especialidades tales c o mo pediatría, ginecobstetricia, cirugía, psiquiatría y ramas de la medicina interna, tales como cardiología, endocrinología y dermatología entre otros abarcando todos los aspectos de la salud y el comportamiento humano.

Al integrar todas estas especialidades médicas, al igual que otras disciplinas aliadas a la salud como nutrición, educación en salud y trabajo social entre otras, los especialistas en medicina de familia están entrenados para brindar servicios de salud y

el cuidado médico a toda la familia independientemente del sexo, edad o condición médica; ofreciendo a sus pacientes un cuidado integral y continuo de excelencia, a la par con los avances tecnológicos y manteniendo el aspecto humanístico de la medicina.

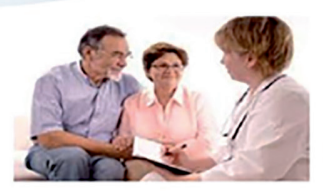

El médico familiar es el especialista que asume la responsabilidad del cuidado integral del paciente y su familia, cubre el desarrollo de todas las etapas del ciclo de vida, nacimiento, crecimiento y desarrollo de la niñez, adolescencia, matrimonio, planificación familiar, contro de embarazo, adultez y vejez, a su vez educa al paciente a mantenerse saludable impartiendo educación para la salud.

17. Crear una Alianza Internacional permanente de Participación y Comunicación Social con la Medicina Familiar. 
Alianza Internacional de Medicina Familiar para la Participación y Comunicación Social de Médicos de familia. CIMF-WONCA. ${ }^{12}$

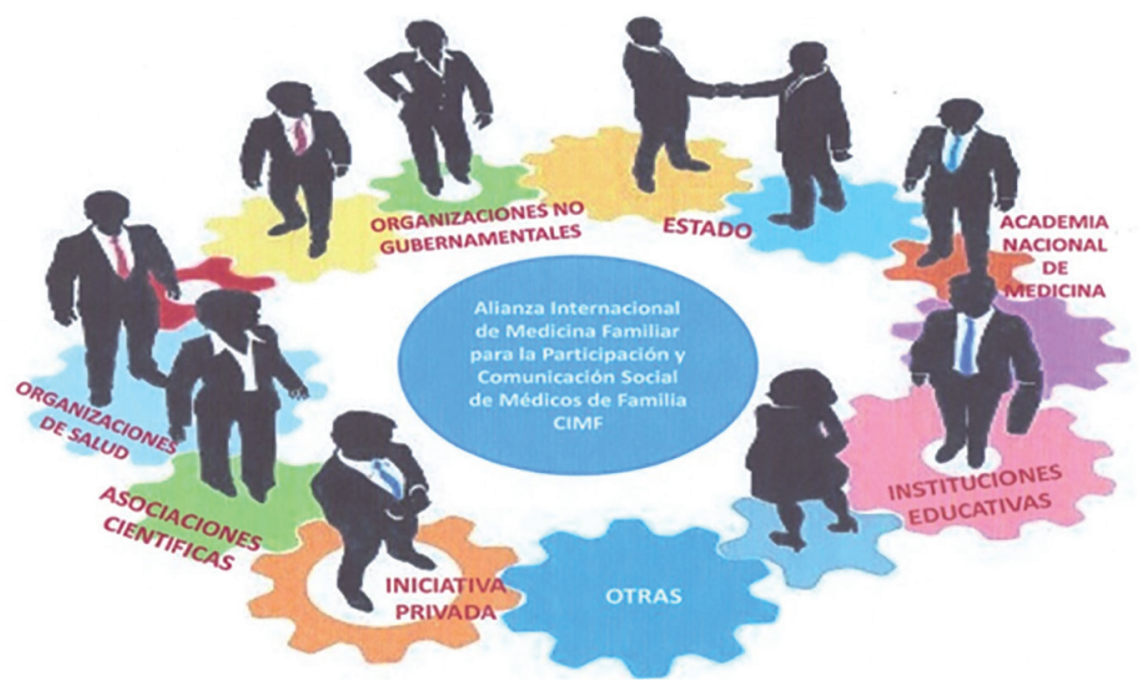

Entidades que deben ser parte de esta ALIANZA:

Sociedades y Asociaciones de Medicina Familiar de Iberoamerica

Fundación Venezolana de Medicina Familiar, FUNVEMEFA

Unidad de Cuidados Primarios, UCP. Venezuela

Centro Integral de la Familia, CIF. Venezuela

Fundación Agenda Ciudadana, Puerto Rico

Organizaciones no Gubernamentales

Movimiento de Salud de los Pueblos

Academia Nacional de la Medicina

Organizaciones de Salud Publica

Alianza para la Salud del Pueblo

Asociaciones Cientificas

Rescarven. Venezuela.

Iniciativa Privada

Universidades 


\section{Referencias}

1. Declaración de Alma ATA. Conferencia Internacional sobre Atención Primaria de Salud, Alma-Ata, URSS, 6-12 de septiembre de 1978. http://www.paho.org/spanish/dd/pin/alma-ata_declaracion.htm

2. Arias Liliana, Brandt Cesar, Freifer Sandra, Fernandez Miguel Angel. Perfil del Médico de Familia, Santiago de Cali, Colombia, mayo de 2010.

3. Confederación Iberoamericana de Medicina Familiar (Wonca Iberoamericana-CIMF). Declaración de Sevilla (España),

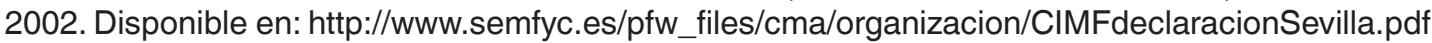

4. CISAS - Centro de Información y Servicios de Asesor en Salud (Centro de Información y Servicios de Asesoría en Salud). Disponible en: http://www.cisa.org.ni/

5. Confederación Iberoamericana de Medicina Familiar (Wonca Iberoamericana-CIMF). Compromiso de Santiago de Chile. II Cumbre de Medicina Familiar, 2005. Médico de Familia, 2005;13(2):11-12.

6. Alianza de Salud para el Pueblo, Declaración de principios y propuestas, 8 de septiembre, 2012.

7. Confederación Iberoamericana de Medicina Familiar (Wonca Iberoamericana-CIMF). Carta de Fortaleza (Brasil), 2008. Rev bras med fam comunidade, 2011;6(supl 1):49-51.

8. Lucha de los pueblos por su salud y liberación en América Latina: Una perspectiva histórica. David Werner, CuencaEcuador, Octubre 2013.

9. Confederación Iberoamericana de Medicina Familiar (Wonca Iberoamericana-CIMF). Carta de la Asunción (Paraguay), 2011. Disponible en: http://www.semfyc.es/pfw_files/cma/organizacion/CIMFcartaAsuncion.pdf

10. Declaración del Movimiento de Salud de los Pueblos de América Latina y el Caribe; http://www.phmovement.org/es/ node/8199.I

11. Patient Centered Medical Home; http://pcmh.ahrq.gov/

12. Werner, David B. and Bill Bower, Aprendiendo a Promover la Salud, Centro de Estudios Educativos, A.C. México. 1984, http://www.redmujeres.org/biblioteca\%20diginal/promocion_salud.pdf 